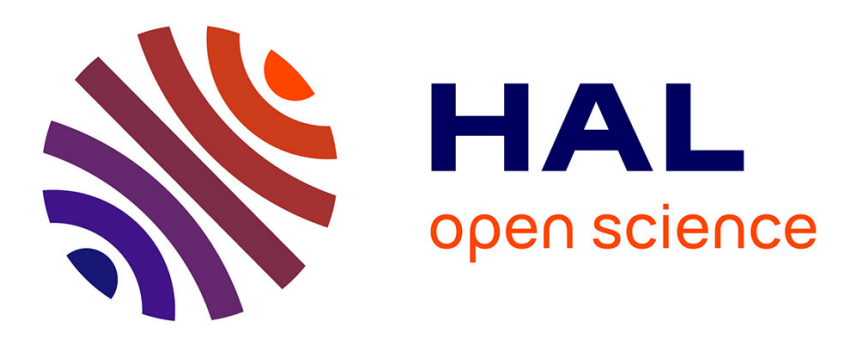

\title{
Validation of a vibration and electric model of honeycomb panels equiped with piezoelectric patch actuators
}

Etienne Balmès, Corine Florens

\section{- To cite this version:}

Etienne Balmès, Corine Florens. Validation of a vibration and electric model of honeycomb panels equiped with piezoelectric patch actuators. Revue des composites et des matériaux avancés = Journal of Composite and Advanced Materials, 2009, 19 (3), pp.319-338. hal-00484000

\section{HAL Id: hal-00484000 \\ https://hal.science/hal-00484000}

Submitted on 17 May 2010

HAL is a multi-disciplinary open access archive for the deposit and dissemination of scientific research documents, whether they are published or not. The documents may come from teaching and research institutions in France or abroad, or from public or private research centers.
L'archive ouverte pluridisciplinaire HAL, est destinée au dépôt et à la diffusion de documents scientifiques de niveau recherche, publiés ou non, émanant des établissements d'enseignement et de recherche français ou étrangers, des laboratoires publics ou privés. 


\title{
Validation of a vibration and electric model of honeycomb panels equiped with piezoelectric patch actuators
}

\author{
Etienne Balmes* - Corine Florens** \\ * Arts et Metiers ParisTech (LMSP) \\ 151 Bld de l'Hopital, 75013 Paris \\ etienne.balmes@paris.ensam.fr \\ ** ONERA DADS, 92322 Chatillon Cedex \\ Ecole Centrale Paris, MSSMat, 92295 Chatenay Malabry
}

\begin{abstract}
Active control has often been considered for low frequency noise control of aircraft or helicopter trim panels. The present study proposes a methodology for numerical homogeneization of honeycombs and shell models of piezoelectric patches. These models are validated experimentally. The considered honeycomb is shown to be significantly viscoelastic and local bending effects are shown to limit achievable active control performance. Finally modeling strategies for the integration of the model in a numerical design process are discussed.

RÉSUMÉ. Le contrôle actif à souvent été considéré pour la maitrise du bruit basse fréquence des panneaux d'habillage des avions et hélicoptères. Dans le cas de panneaux en nid d'abeille, les résultats n'ont pas toujours été à la hauteur des attentes. L'étude présentée introduit un modèle détaillé de panneau en nid d'abeille équipé de patchs piézoélectriques et valide ce modèle expérimentalement. L'utilisation de modes périodiques permet une analyse détaillée de l'influence des constituants dans le comportement vibratoire du nid d'abeille. Les effets viscoélastiques présents pour le Nomex sont par ailleurs mis en évidence. Du point de vue électrique, on montre que la flexibilité résiduelle induit une cloque, flexion très localisée des peaux. La performance atteignable par le contrôle actif est donc limitée. Finalement, des stratégies pour l'intégration du modèle proposé dans un processus de conception sont discutées.
\end{abstract}

KEYWORDS: active control/ honeycomb/ test-analysis correlation

MOTS-CLÉS : contrôle actif/ nid d'abeille/ corrélation calcul-essai

RCMA - 19/2009. Composites multifonctionnels et réactifs, pages 319 à 337 


\section{Introduction}

Reduction of noise transmission inside cabins, for improved passenger comfort, is an important concern for the aircraft industry. Usually trim panels are made of honeycomb sandwich composite. Those panels have a high strength to weight ratio, but acoustical properties have to be improved by complementary treatments. Passive treatments are efficient for high frequencies. Active sound and vibration control is often considered as a solution to reduce residual noise for low and medium frequencies.

While active control of honeycomb panels has been the object of various studies (Bihan et al., Avril 2005), detailed modeling of the panels equipped with piezoelectric patches was not performed. In general, such modeling seems necessary to achieve a consistent design methodology and was thus the general objective of this study.

Section 2 discusses honeycomb modeling using the classical assembly of three models : two skins, modeled as composite plates, and a core, modeled as an equivalent orthotropic volume. A numerical homogeneization methodology based on the correlation of periodic bending mode frequencies is introduced to evaluate the effective parameters of the orthotropic material. This methodology is then used to analyze factors influencing the effective parameters.

Section 3 introduces a piezoelectric shell element and discusses methodologies for numerical resolution of the coupled mechanical/electrical problem.

Experimental validations of the proposed models are discussed in section 4. The Nomex honeycomb considered in the test is shown to be significantly viscoelastic. The identified frequency dependent constitutive law is sufficient to accurately predict transfers of the active panel. Local bending effects in the honeycomb skins are then studied in detail since they are a factor limiting the performance that can be achieved in active control.

Finally section 5 discusses practical constraints linked to the use of the proposed model in a design phase.

\section{Models of honeycomb panels}

\subsection{Equivalent models}

When modeling honeycomb panels, the use of detailed 3D models where each of the walls of the honeycomb is modeled can rapidly become inaccessible. The classical approach is thus to build an equivalent representation with skins modeled as shells and the honeycomb modeled using an orthotropic volume. A wide range of equivalent formulas giving the effective properties of the orthotropic material as function of the cell properties can be found in the literature (Gibson et al., 1997; Grédiac, 1993; Noor et al., 1997; Hohe et al., 2001). 
The first objective of the present work is to propose a systematic approach to evaluate the effective (or equivalent) properties based on a detailed FE model of the honeycomb.

To demonstrate the approach, one considers the mesh shown left in figure 1. This model is meant to represent physical reality (geometry, thickness of various walls, material...) as closely as possible, with the intention of minimizing the modeling errors. It will be used as reference to estimate honeycomb properties starting from material and geometric considerations.
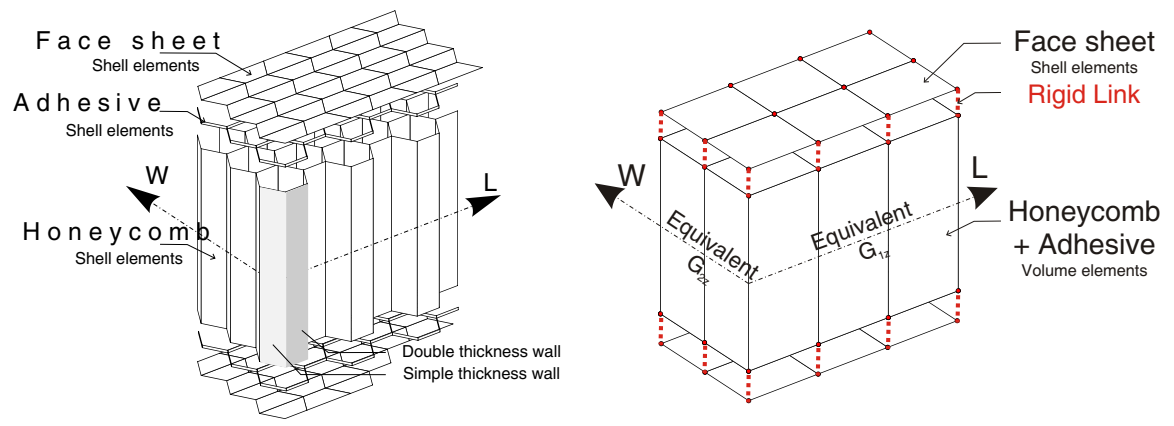

Figure 1. Honeycomb representative volume. Left : Detailed 3D model (3D). Right : shell volume shell model (SVS)

The geometry of the glue is shown in figure 2. Rather than introducing a detailed geometry, the 3D model, considered here, privileges a functionally equivalent representation as a small plate extending from the skin neutral fiber to its surface. The explicit representation of glue elements thus allows a meshing of the honeycomb cell walls and face-sheets that is compatible and strictly respects the geometry. Functional values of shear and bending stiffness can then be easily related to the glue modulus $E_{g}$ and shell element thickness $t_{g}$.

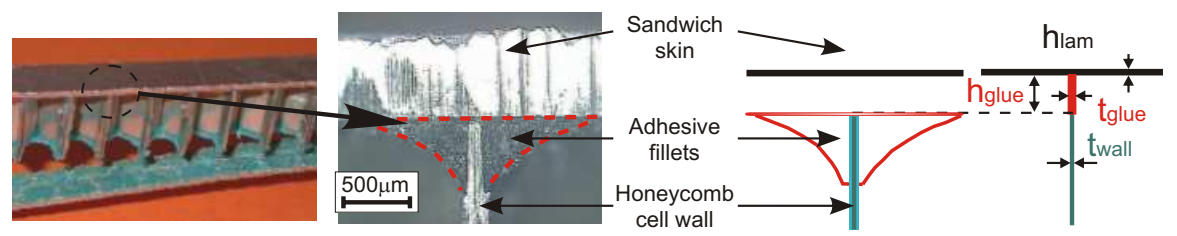

Figure 2. Modeling of adhesive fillet

The classical equivalent model for large panels uses the shell/volume/shell (SVS) layout shown right in figure 1 . The sandwich face sheets are modeled by isotropic 
shell, for metallic skin such as aluminum, or multilayered composite laminate, for composite skin such as carbon fiber reinforced composites. As in most of the literature, Mindlin formulations and Classical Laminated Plate Theory (Jones, 1975; Berthelot, 1992) is assumed to be sufficient. For the simulations shown, the SDT (Balmes et al., 2009) implementation of MITC4 composite plate/shell elements with shear correction is used. To simplify handling, the face sheets are normally meshed to be compatible with the underlying core models. In the case of detailed 3D models, one thus uses hexagonal shapes composed of two quadrangular elements, while in the SVS model regular rectangles are used.

\subsection{Determination of effective parameters using periodic modes}

Homogeneization techniques determine effective properties of the homogenized model by comparing various properties (static deflections, strain energies for particular deformations, ...). The approach retained here is to compare the frequencies of bending modes obtained with periodicity conditions that are equivalent for both models shown in figure 1 and to update the effective parameters of the SVS model to match the frequencies of the detailed 3D model. This is a numerical homogeneization procedure since one uses a detailed 3D FEM model as reference and a SVS model to determine the effective parameters. The update can be performed for all wavelengths and the range of stability of the parameter is a useful result of the study.

While periodic modes can be computed using a long beam sample and simple conditions of equal displacement at the wavelength (Florens et al., 2006), this approach is costly. These modes can however be computed much more efficiently using Fourier theory as detailed in (Clouteau et al., 2005) for the periodic structures and (Sternchüss, A. and Balmes, E. and Jean, P. and Lombard, JP., 2008) for cyclic symmetry.

This section summarizes the theory underlying periodic mode computations. One considers a model whose properties are spatially periodic. For a physical response, known at regularly spaced positions $n \Delta x$, one can compute its Fourier transform

$$
U(\kappa)=\sum_{n=-\infty}^{+\infty} u(n \Delta x) e^{-j \kappa n \Delta x}
$$

$U(\kappa)$ is a complex shape defined on the mesh of the repeated cell. One actually uses two cells to represent the real and imaginary parts of $U$. The wave number $\kappa$ varies in the $\left[0, \frac{2 \pi}{\Delta x}\right]$ interval (or any interval of the same length, since $U(\kappa)$ is periodic in the wavelength domain).

Periodic modes can be shown to be mono-harmonic (to involve a single $U(\kappa)$ ). As a result one has

$$
u(n \Delta x)=\operatorname{Re}\left(U(\kappa) e^{j n(\kappa \Delta x)}\right)
$$


This property can be used to recover motion on any cell based on known values on the first one. Using the continuity condition linked to the fact that $u_{\text {left }}(n \Delta x)=$ $u_{\text {right }}((n-1) \Delta x)$ and observation matrices $c_{L}, c_{R}$ to extract the edge motion, one can write $\left[c_{L}\right]\{U(\kappa)\}=\left[c_{R}\right]\{U(\kappa)\} e^{-j(\kappa \Delta x)}$. Hence in the real/imaginary format, one has the constraint equation on nodes of the cell edge

$$
\left[\begin{array}{cc}
{\left[c_{l}\right]-\cos (\kappa \Delta x)\left[c_{r}\right]} & -\sin (\kappa \Delta x)\left[c_{r}\right] \\
\sin (\kappa \Delta x)\left[c_{r}\right] & {\left[c_{l}\right]-\cos (\kappa \Delta x)\left[c_{r}\right]}
\end{array}\right]_{2 N_{r} \times 2 N}\left\{\begin{array}{c}
\operatorname{Re}(U) \\
\operatorname{Im}(U)
\end{array}\right\}=0
$$

Since periodic modes are mono-harmonic solutions of wavelength $\pi / \kappa$, one thus solves for

$$
\left[\begin{array}{cc}
K-\omega_{j}^{2} M & 0 \\
0 & K-\omega_{j}^{2} M
\end{array}\right]\left\{\begin{array}{l}
\operatorname{Re}(U) \\
\operatorname{Im}(U)
\end{array}\right\}=0
$$

with [3] verified. Figure 3 illustrates the basic cell and restitution on multiple cells using [2] of modes computed for $1 / \kappa$ equal to 3 and 17 .

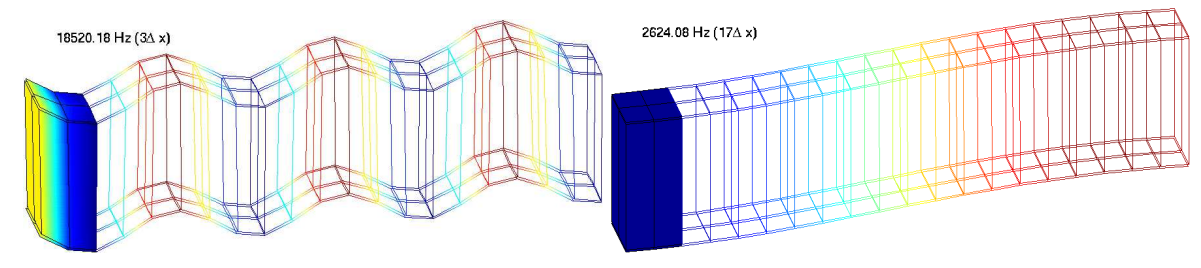

Figure 3. Periodic modes of the SVS model

\subsection{Analysis of properties}

For the detailed 3D model of the nominal honeycomb in this study, figure 4 displays the evolution of the periodic mode frequency as a function of wavelength and the associated energy contributions. $A$ terms refer to membrane/shear effects and $D$ to bending (see (Jones, 1975) and section 3.1)

The plot clearly indicates very different regimes. Above 1000 cell lengths (at low frequencies), the energy is essentially due membrane effects in the skin. This is underlying principle of honeycombs; the core only serves to separate the skins which carry all the load. Between 3 and 100 cell lengths, the core contains a major part of the energy. Its properties are thus critical to properly represent motion with relatively short wavelengths.

For very short wavelengths and very high frequencies, one starts seeing local modes of the skin or local bending of the cell walls. Such modes would be described as surface waves and are not really of interest to the present study. 

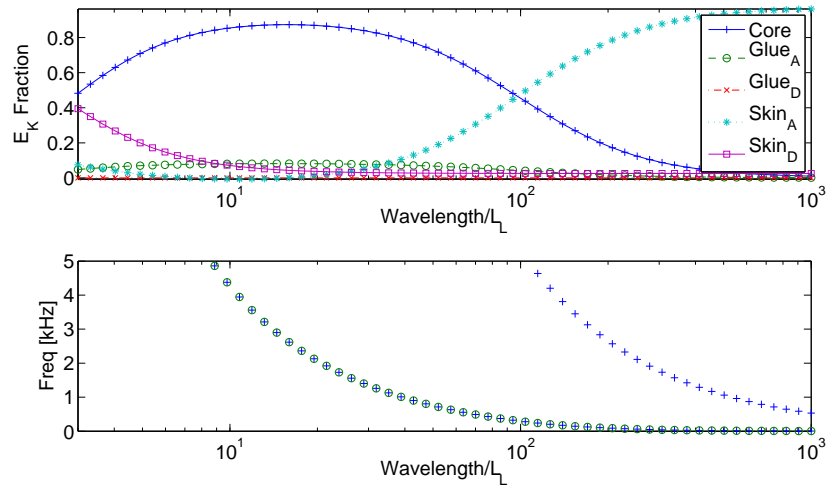

Figure 4. Energy contribution of constituents and frequency of longitudinal periodic modes as a function of wavelength divided by cell length $L_{L}$. Detailed $3 D$ model

For the parameters of figure 4, the shear energy in the glue is above $8 \%$ in the 10 to 50 cell lengths range. Figure 5 illustrates the evolution of energy fractions in the glue layer for varying glue moduli. Since $0.2 \mathrm{GPa}$ is a very realistic value for epoxy it clearly appears that the glue can indeed have a major impact on the short wavelength dynamics.

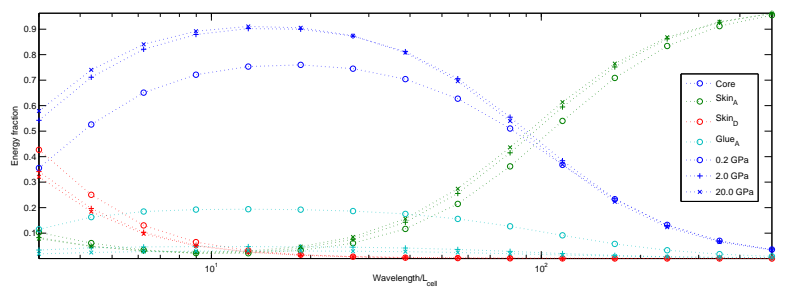

Figure 5. Energy fraction of constituents. Detailed 3D model with 0.2 to $20 \mathrm{GPa}$ glue modulus

Figure 6 shows the same plot for the SVS model. Similar regimes are found and one verifies the classical results that almost all the energy in the core is associated with the $G_{x z}$ shear. 


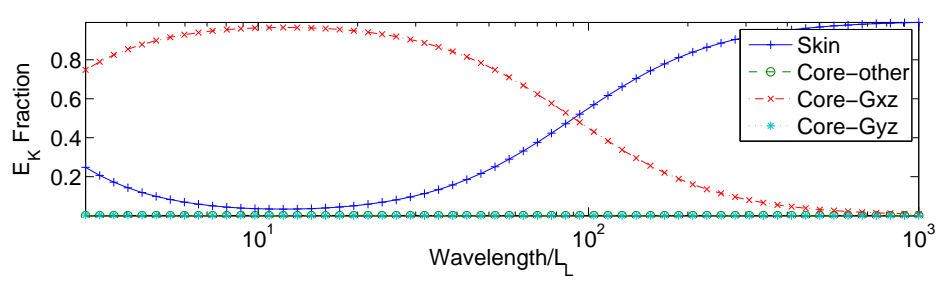

Figure 6. Energy contribution of constituents for first longitudinal periodic mode as a function of wavelength. SVS model

Figure 7 illustrates the proposed homogeneization procedure. One computes, and shows in the top graph, the frequency error on the corresponding first bending mode for multiple values of $G_{x z}$. In the present case, one sees a divergence below $5 \Delta x$. This is associated with the fact that cell wall bending occurs near $10 \mathrm{kHz}$ and becomes the first periodic mode for very short wavelengths.
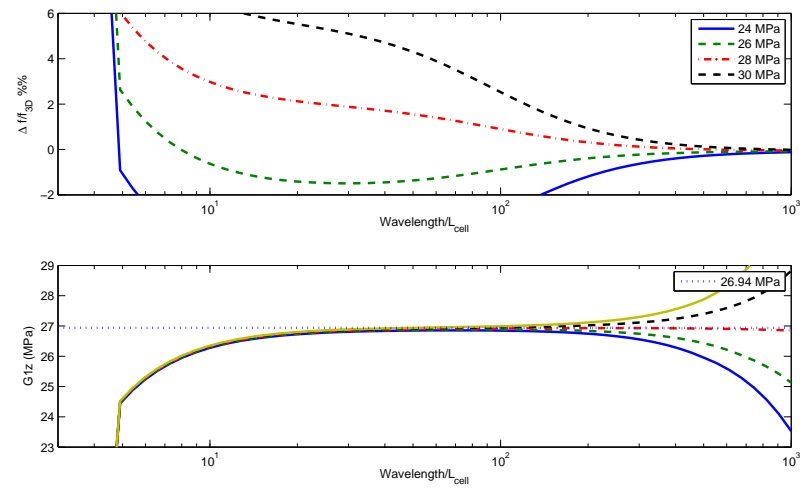

Figure 7. Top: error on frequency of the first periodic bending mode for various values of $G_{x z}$. Bottom: Estimation of $G_{x z}$ associated with no error. Detailed $3 D$ model with 4 by 4 refinement (curves correspond to $+1 \%$ to $0 \%$ mass)

The bottom graph shows the evolution of the $G_{x z}$ value for which the frequency error is null. The mean value is $26.94 \mathrm{MPa}$. The nominal model (top curve) showed an increase of the estimated modulus for large wavelength. Several studies were performed to analyze the source of this discrepancy. The skin models clearly have no impact: a SVS model that has skin elements identical to the 3D model also shows the same decrease. Eventually, it was found that the density of the core is an extremely influent parameter in this range. This is not unexpected since the core has very limited strain energy and thus significant modulus changes are needed to compensate for 
small frequency errors associated with the mass. The nominal model overestimates the mass by $1 \%$. The five curves are evenly spaced in the $1 \%$ to $0 \%$ interval. A nearly constant modulus is thus found for a $0.5 \%$ overestimation of the mass.

In practice, honeycomb bonding procedure clearly induces uncertainties in the mass much larger than $0.5 \%$. The proposed analysis thus motivates the need for experiments evaluating the transverse shear for waves in the 30 to 200 cell length range $(1500 \mathrm{~Hz}$ to $100 \mathrm{~Hz})$.

\section{Model of piezoelectric patch actuators}

\subsection{Laminated piezoelectric plates}

The piezoelectric plate element, implemented in (Balmes et al., 2009) and considered in this work, is based on Classical Laminated Plate Theory (CLPT (Jones, 1975; Berthelot, 1992)) for the mechanical part and the work of Piefort (Piefort, 2001) for the piezoelectric coupling.
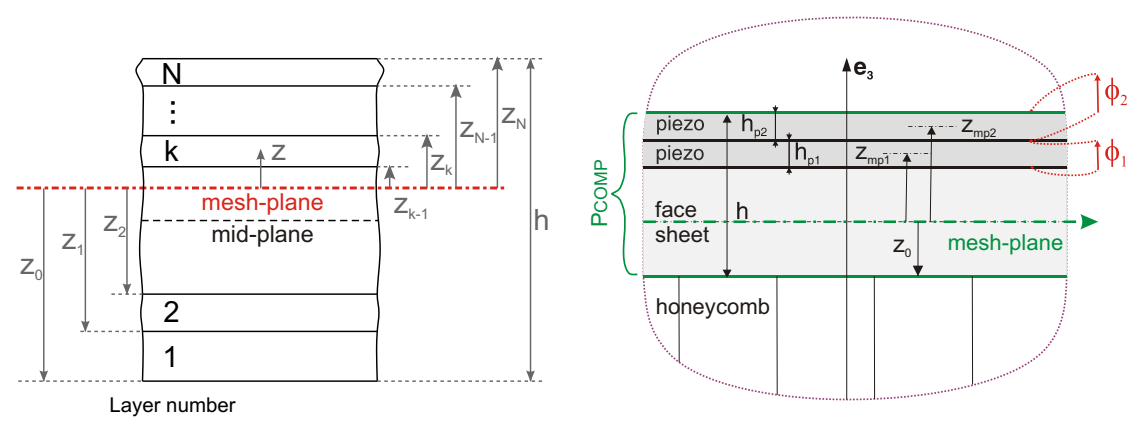

Figure 8. Left Geometry of an N-layered laminate. Right Particularization for a skin with a piezoelectric patch

The global constitutive equations of piezoelectric Mindlin shell are obtained by integration over thickness and are expressed in the global coordination system. The piezoelectric medium has orthotropic mechanic and electric properties; hence the constitutive equations for each layer are related to their orthotropic axes. $\left[R_{S}\right]_{k}$ is the transformation matrix linking the strain in the global coordinate system to the local one, and $\left[R_{T}\right]_{k}^{-1}=\left[R_{S}\right]_{k}^{T}$ is the one linking the stresses in the local coordinate system to the global one. The coupled plate/piezoelectric equations are thus given by 


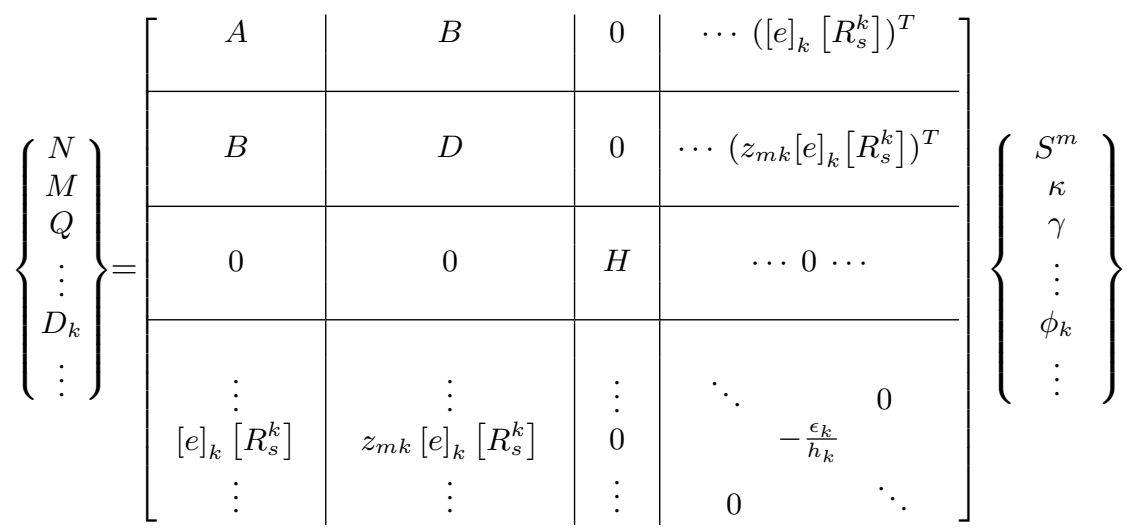

where the constitutive matrices $[A]$ (membrane), $[B]$ (membrane/bending coupling) $[D]$ (bending), $[F]$ (shear) are those found in classical laminated plate theory (see (Jones, 1975)).

It is assumed that the electric field needs only to be known between electrodes and can be described by the difference of potential $\phi_{k}$, leading to an assumed electric field $\vec{E}=-g r a d \phi$. When this field is applied in the direction $\vec{e}_{3}$, the piezoelectric actuator PZT extends in the plane $\left(\vec{e}_{1}, \vec{e}_{2}\right)$. It produces load on the piezoelectric layer (or ply in the CLPT terminology) in the direction $\vec{e}_{1}$ which depends on $e_{31}$ and $\phi$. Because of the offset between the mid-plane of the actuator and the mid-plane of the multilayer laminate, the force induces both membrane and bending loads as will be illustrated in section 4.

In the software implementation, the composite plate is modeled using a classical MITC4 element (Bathe et al., 2003). The piezoelectric coupling part of [5] is numerically integrated using the nominal four point rule on the patch. For the electric part, a single electric DOF is considered for each patch. This DOF is thus common to all elements covered by the electrode. In the present configuration two patches are stacked together thus leading to a model with two electric DOFs. In practice, the wiring is such that these potential are equal or opposite. The elimination of this constraint is done in the model resolution phase.

\subsection{Resolution of system equations}

Once assembled the model takes the form

$$
\left[\begin{array}{cc}
Z_{u u} & K_{u \phi} \\
K_{\phi u} & K_{\phi \phi}
\end{array}\right]\left\{\begin{array}{c}
q_{m e c h} \\
\phi_{k}
\end{array}\right\}=\left\{\begin{array}{c}
F_{m e c h} \\
Q_{k}
\end{array}\right\}
$$

with the classical dynamic stiffness $Z=M s^{2}+K$. When simulating the response, one needs to consider sensor and actuator configurations. 
Piezoelectric patches used as sensors have a charge that remains zero (open circuit mode). One can thus condense the electric DOF. Indeed, one considers an electrostatic behavior (no $M_{\phi \phi}$ or $M_{\phi u}$ ) and assumes $Q_{k}=0$, one thus has

$$
\left\{\begin{array}{c}
\left\{q_{\text {mech }}\right\} \\
\phi_{k}
\end{array}\right\}=\left[\begin{array}{c}
I \\
-\left[K_{\phi \phi}\right]^{-1}\left[K_{\phi u}\right]
\end{array}\right]\{q\}_{\text {mech }}=[T]\left\{q_{\text {mech }}\right\}
$$

This relation can be used to eliminate the electric contribution from the system equations which become

$$
[T]^{T}[Z][T]\left\{q_{\text {mech }}\right\}=[T]^{T}\{F\}
$$

From this condensation it clearly appears that a patch used as sensor induces a shift of frequencies form a configuration without piezoelectric coupling.

Piezoelectric patches used as actuators have a difference of potential $\phi_{k}$ that is enforced. One can thus use the electric part of [6] to determine the charge

$$
Q_{k}=\left[\begin{array}{ll}
K_{\phi u} & K_{\phi \phi}
\end{array}\right]\left\{\begin{array}{c}
q_{m e c h} \\
\phi_{k}
\end{array}\right\}
$$

and consider the system equations with no piezoelectric coupling and an electric load

$$
Z_{u u} q_{m e c h}=F_{m e c h}-K_{u \phi} \phi_{k}
$$

It clearly appears that the dynamic stiffness of this configuration is that of the purely mechanical model with no piezoelectric coupling. The modes of $Z_{u u}$ correspond to the case with $\phi_{k}$ set to zero. In other words, the actuator configuration leads to closed circuit modes.

In the resolution process implemented in (Balmes et al., 2009), electrodes are declared to be either open or closed circuit which allows a condensation or elimination of electrical DOFs before computation of modes and static correction for inputs (Balmes, 1997-2007), which are used to build the state-space model. The inclusion of the static correction is shown to be critical in the next section.

\section{Experimental validation}

\subsection{Test setup}

Multiple modal tests were performed at Ecole Centrale Paris (MSSMat) and KTH Stockholm University (MWL). The test beams external dimensions are $900 \times 45 \times$ $21 \mathrm{~mm}$ (approximately $109 \times 5$ cells). Configurations with $0.6 \mathrm{~mm}$ aluminimum and $1 \mathrm{~mm}$ carbon laminates were tested. The core is a $19 \mathrm{~mm}$ thick Nomex paper honeycomb. The carbon skin beam was equiped with 3 QuickPack piezoelectric patches 
detailed in figure 9. Excitation was performed using a shaker or the piezoelectric patches.

Measurements over the full length of the beam were performed to obtain global modeshapes correlation. When local effects were shown, a second test was performed using a dense mesh of measurement points located over the patch.
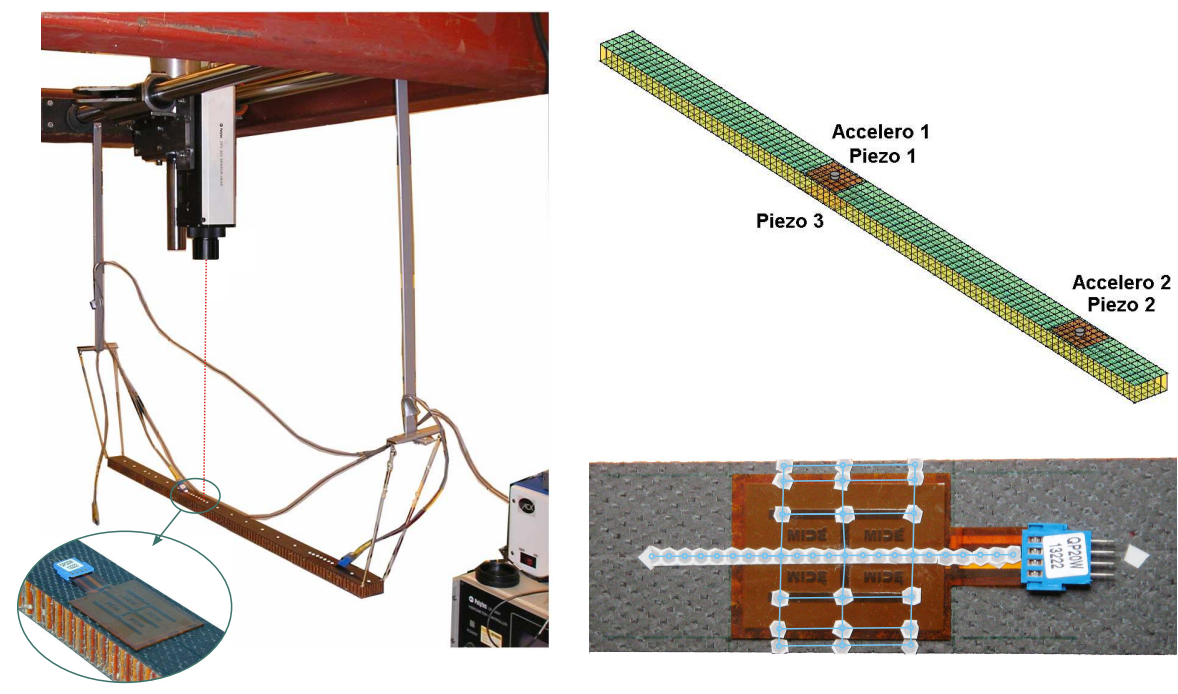

Figure 9. Experimental Setup. Left : full beam measurements. Top right, piezo patch configuration. Bottom right: sensor location of zoom on patch

\subsection{Viscoelastic properties of Nomex}

As shown in figure 10 the Nomex honeycomb and/or glue considered in this study show significant viscoelastic behavior. One indeed clearly sees a evolution of stiffness and loss factor with temperature.

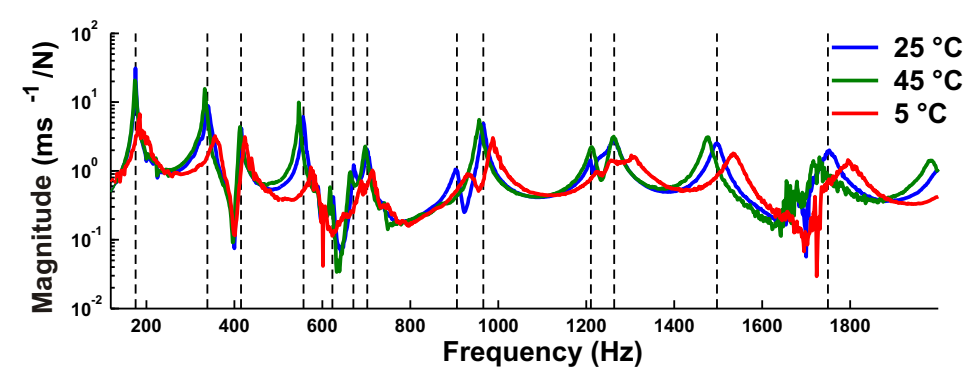

Figure 10. Frequency Response for various temperatures. Aluminum Nomex honeycomb (Florens et al., 2006) 
Using the SVS model of the tested beam, an updating of the G1z modulus was performed for each mode in order to find the value minimizing the error on modal frequencies. In figure 11, the resulting modulus shows the increase with frequency and decreasing slope classically found for viscoelastic materials.
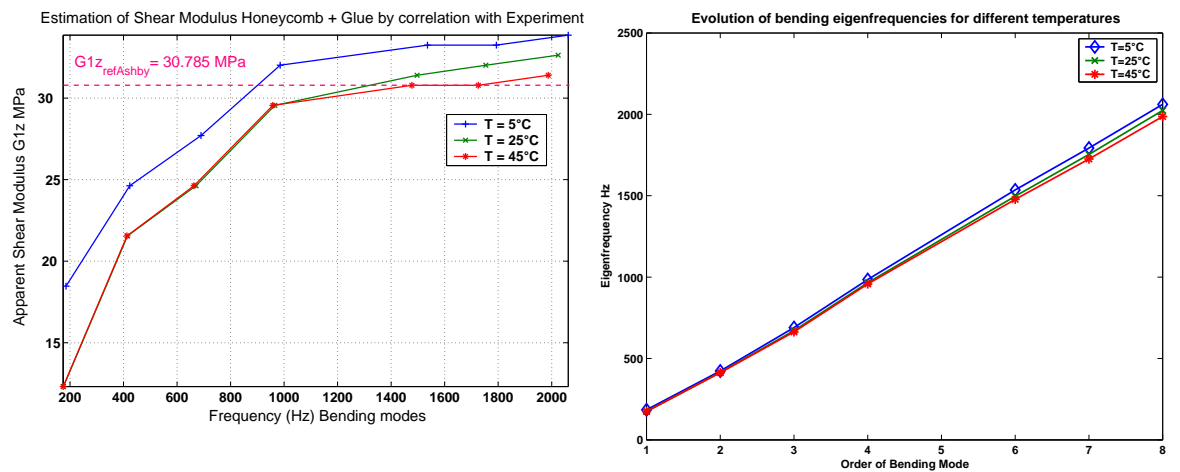

Figure 11. Evolution of bending eigenfrequencies for different temperatures. Equivalent shear modulus G1z of honeycomb + glue core as a function of frequency and temperature

The experimental conditions are clearly not sufficient for a complete validation of the $G_{1 z}$ frequency/temperature dependence, but the trend is clearly shown and was found to be sufficient for the purposes of this study. Figure 12 indeed demonstrates that excellent test/analysis correlation is achieved, provided that the frequency dependence of the honeycomb properties is taken into account.

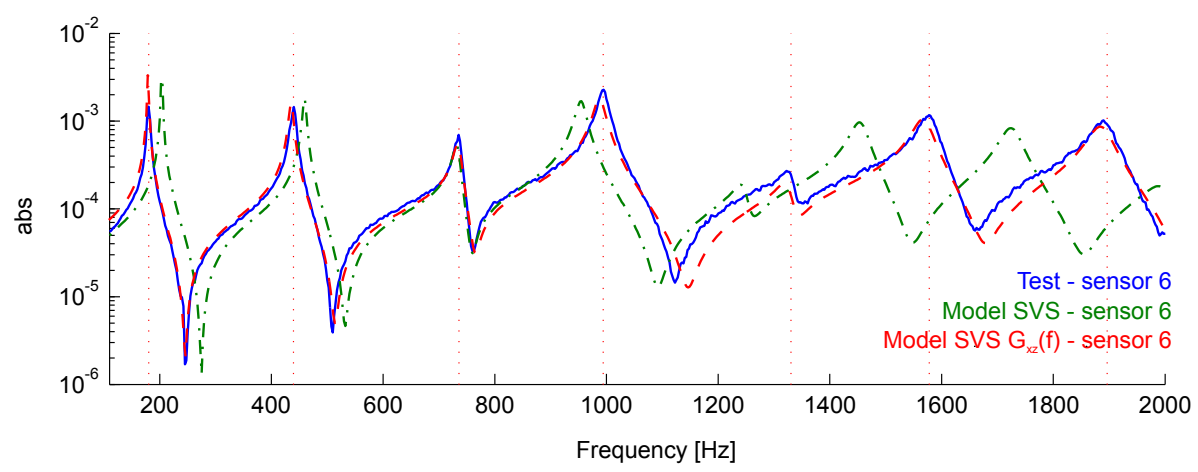

Figure 12. Piezo voltage to translation transfer on a carbon/Nomex honeycomb beam (Piezo 1 actuator - Laser sensor 6) 


\subsection{Piezoelectric patch effectiveness}

Another series of experiments was focused on obtaining a clear understanding of the limitations of the actuation mechanism obtained by placing patches on the skin.

The problematic shape is illustrated by simulation results shown in figure 13. It is well known that patches generate loads that correspond to a combination of in plane membrane traction and moments applied on the edges, as shown in the figure. The use of a two layer patch in the QuickPack is meant to allow users select a preferred actuation mechanism depending on whether the two applied voltages $\phi_{1}, \phi_{2}$ have equal or opposite polarity. In the considered setup, the polarity favoring membrane loading is used. The objective is thus to generate an elongation of the skin and thus a global bending of the beam.

The shape, shown in figure 13, clearly indicates a poor actuation mechanism. While bending of the overall beam is achieved, the dominant effect at this frequency is a very local bending of the skin. The effect is even large enough to generate a local bending of the opposite skin. With such behavior, the patch is more effective as a local loudspeaker than as a actuator on global bending modes. The result being far from obvious and not documented in the literature, detailed tests were performed.
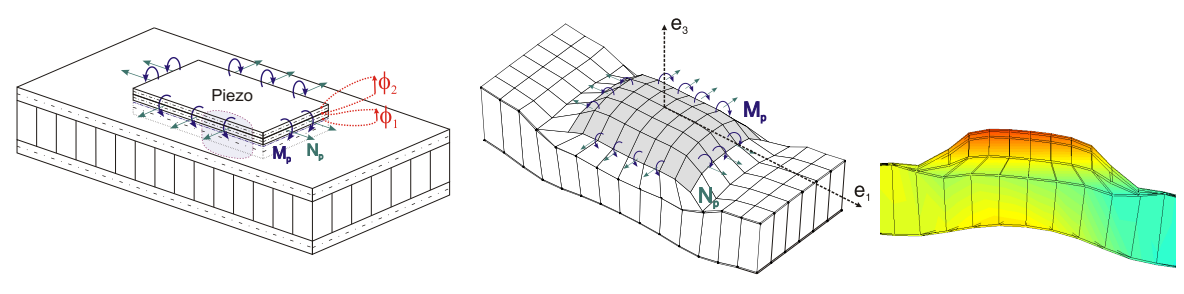

Figure 13. Left : Patch configuration. Center : equivalent applied loads. Right : simulated blister of piezoelectric actuator

First, it is important to understand that the localized bending effect cannot be seen in the shape of the low frequency modes. To illustrate the point, figures 14 and 15 compare forced responses, computed and simulated at and around the second mode resonance. The test/analysis correlation is very good and clearly demonstrates that the modeshape does not show the blister shape, while off-resonance responses show it clearly. 
332 RCMA - 19/2009. Composites multifonctionnels et réactifs

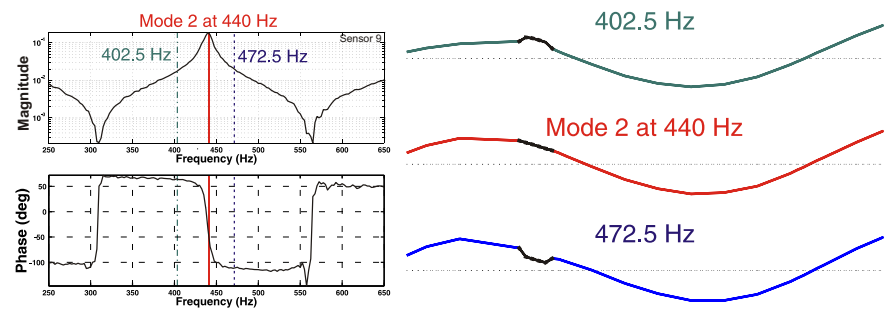

Figure 14. Test of second mode. Carbon/Nomex honeycomb core beam actuated by patch 1. Left : response in the middle of the patch. Right : shape before, at, and after resonance

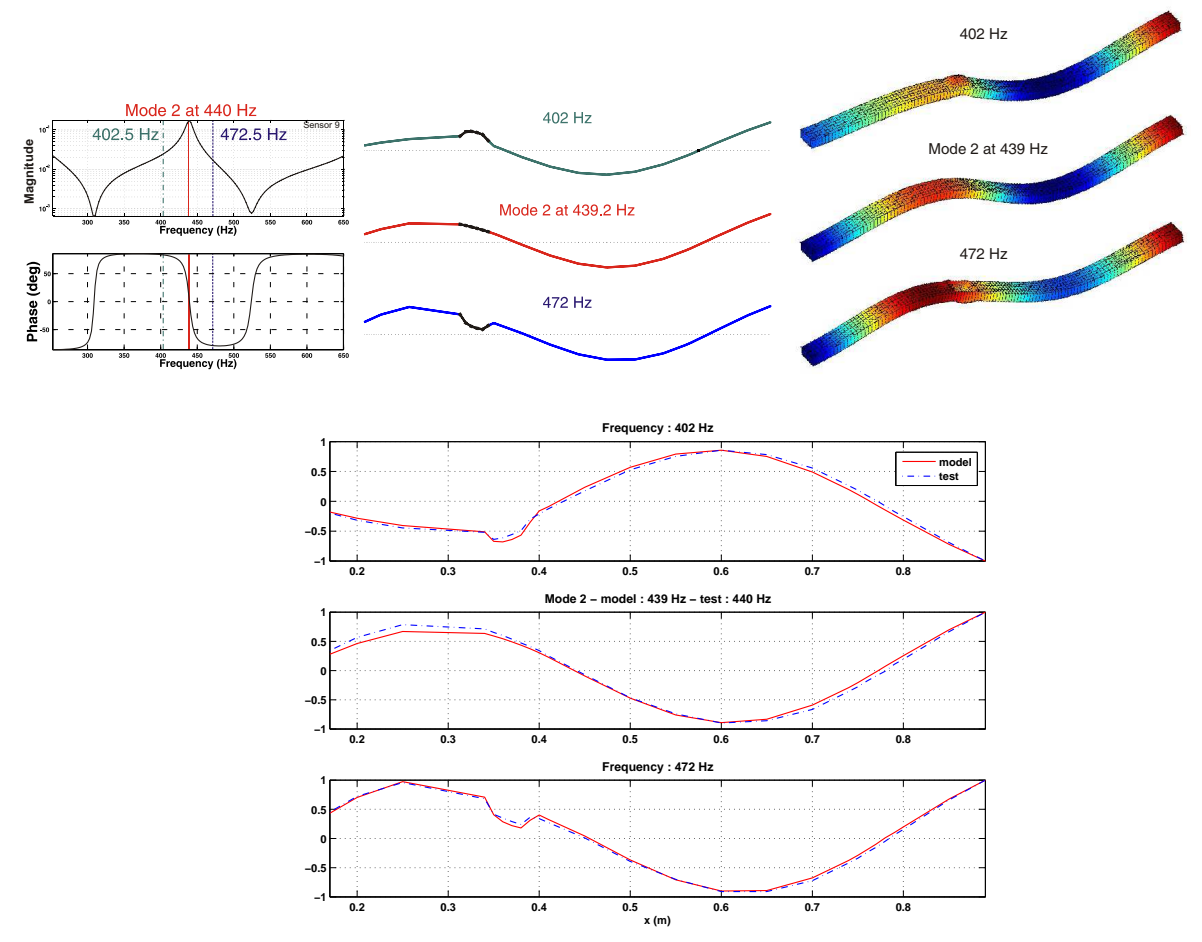

Figure 15. Simulation near mode 2 of the carbon/Nomex honeycomb core beam actuated by the piezoelectric patch 1. Left : response at the middle of the patch. Right: Shape before, at, and after resonance. Bottom : test analysis/correlation

To establish the test/analysis correlation more firmly, the density of measurements was refined on the patch in both $\mathrm{x}$ and $\mathrm{y}$ directions. The quantitative comparison of the 
local patch deflection in $\mathrm{x}$ and $\mathrm{y}$ directions are presented in figure 16. Measurement errors are visible, especially in $\mathrm{x}$ direction because the sensors were really close, only $3.8 \mathrm{~mm}$ between each reflector. Despite the measurement error, the shapes are very well correlated. The FE model presented is thus clearly validated.
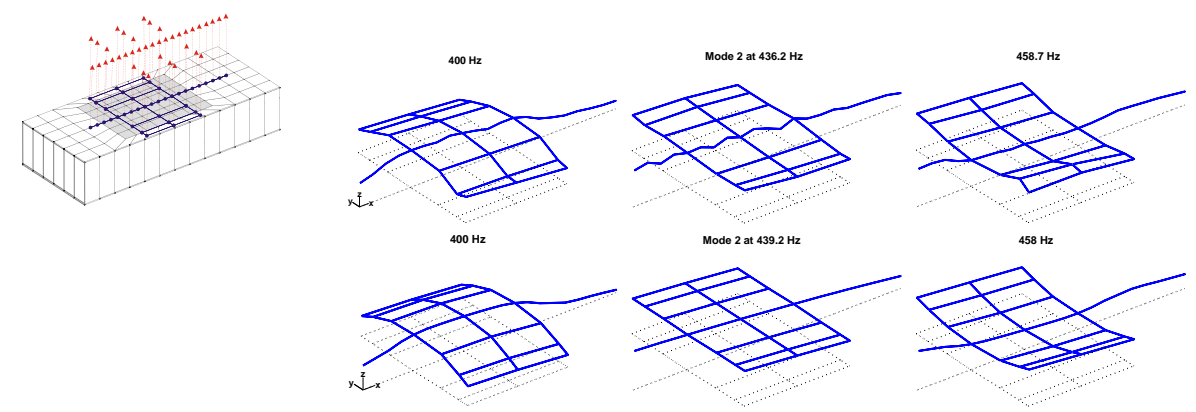

Figure 16. Test (top) and simulation (bottom): before resonance at $400 \mathrm{~Hz}$, at resonance, and after resonance at $458 \mathrm{~Hz}$

For vibration applications, the response is a linear combination of the modal contributions and a quasi-static effect, often called residual flexibility (Craig, 1987). The blister shape is the deformation generated by the application of a static voltage on the patch. The test detailed in this section illustrated the fact that the residual flexibility cannot be neglected outside resonances. This effect has an obvious limiting effect on the ability to control noise radiated by the panel since control of the modes will generate a local noise source even on the skin opposite to the patch.

\section{Strategies for integrating piezos into FE models}

Having validated a FE model, this section focuses on the practical implementation of the modeling strategy. The model considered in previous sections implies significant adaptations. As shown in figure 17/a, the mesh is adapted in the vicinity of the patch and two composite properties are considered. One for the standard honeycomb skin (shown in blue in the figure) and one for the patch which includes multiple layers for the skin plus two layers for the patch, as shown in figure 8 .

This approach is fine for a small beam model and if only few configurations of patch locations are considered. Indeed, the adaptation of the mesh under the patches would be quite time consuming for a large panel where multiple patch configurations would need to be tested. Furthermore, the use of viscoelastic materials in the glue, considered by many in the literature, implies the use of a multi-layered model with the glue represented as a volume element. 
The idea tested in this work is thus to use separate elements to model the skin and the piezoelectric patch, with linear constraints used to account for the offset between the two. The shear stiffness of the glue layer $(G / h)$ is deemed sufficiently high to suppose perfect bonding and thus omit glue modeling.

An automated procedure was introduced to mesh the patch. Based on a given rectangular shape, skin elements strictly under the patch are projected to the patch mid-surface. The boundary between the projected elements and the edge of the reference rectangle is then meshed automatically. The resulting model is shown in figure $17 / \mathrm{c}$. This approach is deemed more acceptable than an adaptation of the honeycomb mesh because it only affects a small part of the model and would be adapted for the reuse of modes of the nominal panel.

As an intermediate validation, a model with two layers (skin and piezo) but an adapted underlying mesh is also considered and shown in 17/b.

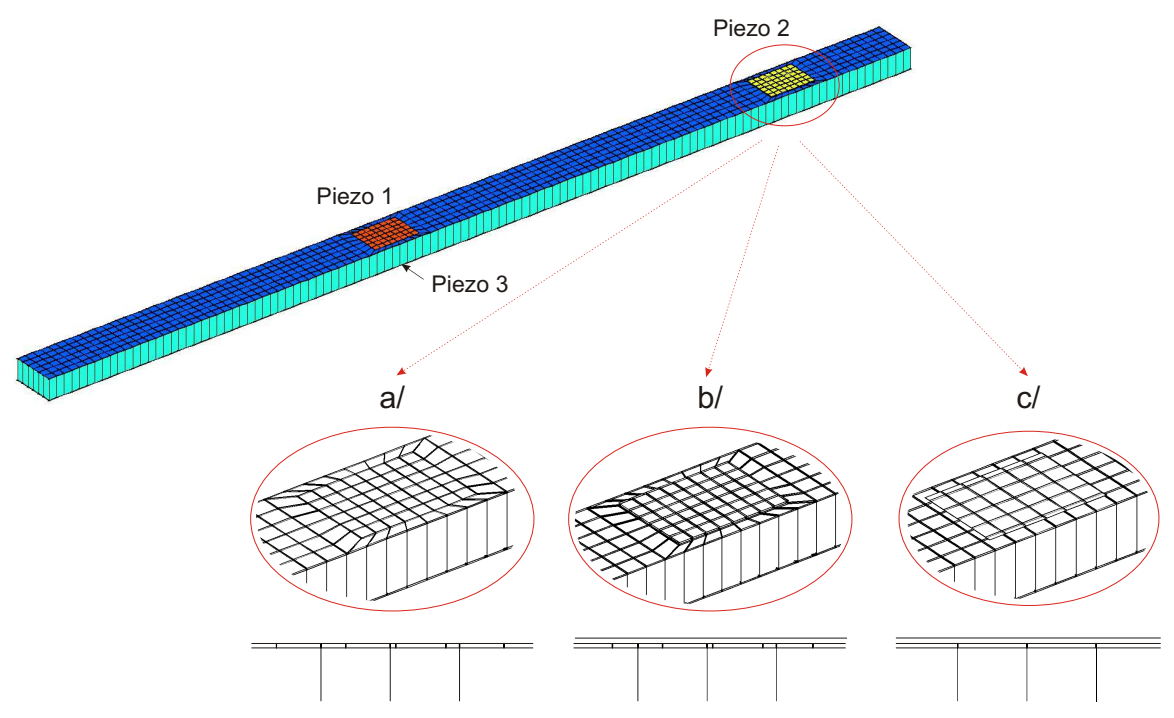

Figure 17. Piezoelectric patch model strategy: a/ adapted meshing and integrated patch, $b /$ adapted meshing and 2 layers (patch and face sheet), c/ non-adapted meshing and 2 layers

To compare these models, electric transfers are shown in figure 18 and piezo to laser transfers in figure 19. For a cross transfer from voltage input on piezo 2 to voltage output on piezo 1, the responses are almost perfectly superposed. For the

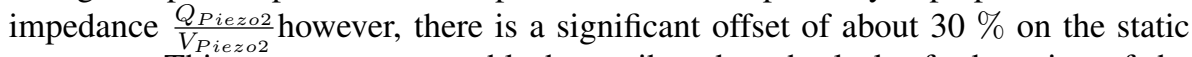
response. This error can reasonably be attributed to the lack of adaptation of the mesh on the patch edges. This is illustrated in figure 20 which shows the quasistatic shaped generated by the actuation (since the structure is free floating, one shows 
the deformation at $1 \mathrm{~Hz}$ which avoids problems linked to rigid body modes). The deformations clearly show that the blister shape under the patch cannot be perfectly reproduced due to the lack of mesh adaptation.
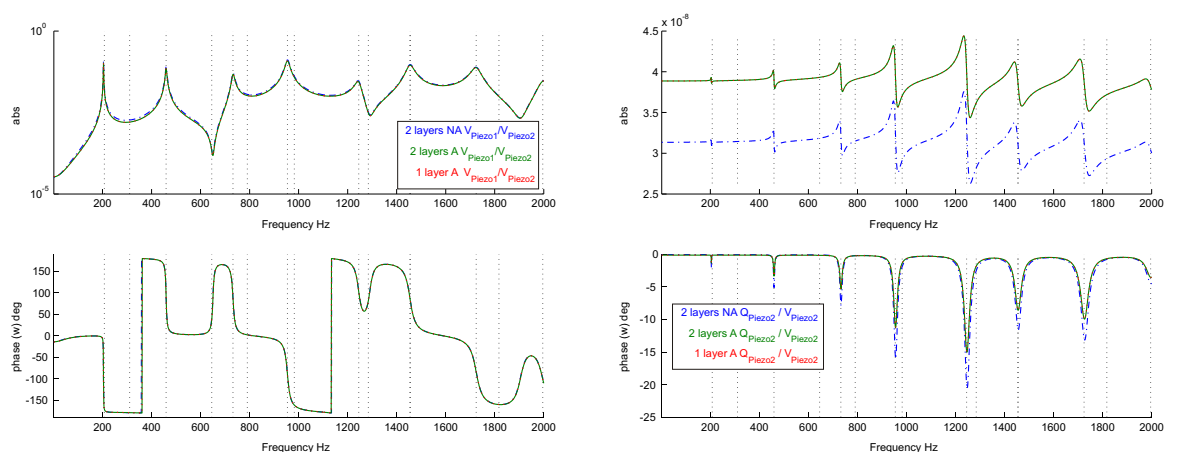

Figure 18. Electric transfers for the 3 model strategies. Input is piezo 2 used as voltage driven actuator. Left piezo 1 used as voltage sensor. Right Charge generated on piezo 2
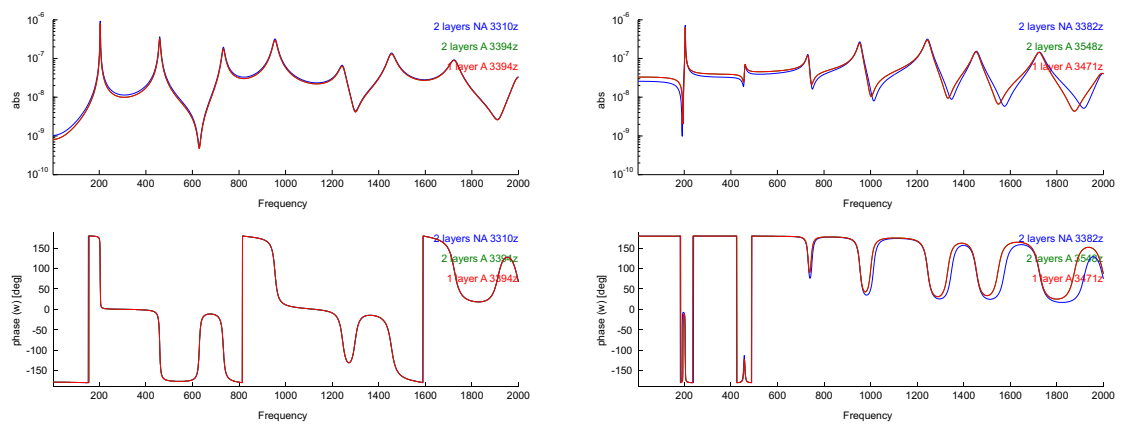

Figure 19. Piezo to laser sensor transfer
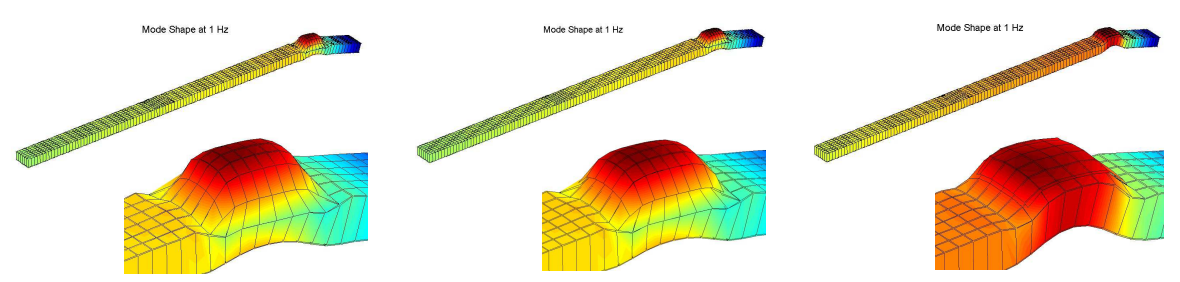

Figure 20. Shape at $1 \mathrm{~Hz}$ for the 3 model strategies of the piezoelectric actuator (Piezo2) 
The proposed strategy using multiple-elements through the thickness is thus limited by the ability to reproduce local effects under the patch. The local nature of this effect is clearly considered a design flaw of the considered configuration. With better designs, the patch would induce bending with much greater wave length and the mesh adaptation would probably be not as necessary.

\section{Conclusion}

Modeling techniques for honeycomb panels equipped with piezoelectric patches are very well correlated with experiments.

For honeycomb models, the classical shell/volume/shell model is well suited for all but very short wavelengths. The numerical homogeneization procedure that was implemented is very efficient and shows that constituent properties, that are not very accurately known (glue modulus, weight and geometry), have a significant impact. Analytic formulas to estimate the orthotropic material properties can thus be used as a starting guess, but should be corrected by correlation with experiments. In the particular case of Nomex honeycomb, the viscoelastic behavior cannot be neglected.

For the piezoelectric patch models, the particular configuration studied shows very strong local effects in the skins, which would give poor results for applications in an active control experiment. Better configurations would imply changing the relative skin/core stiffness. This can be achieved for other skin/core and skin/patch thickness ratios and possibly by introducing local modifications of the skin thickness around the patch. Automating the search for such efficient configurations is a clear perspective of the work presented here.

\section{References}

Balmes E., Methods for vibration design and validation, Course notes Ecole Centrale Paris, 1997-2007.

Balmes E., Bianchi J., Leclère J., Structural Dynamics Toolbox 6.2 (for use with MATLAB), SDTools, Paris, France, www.sdtools.com, Sep, 2009.

Bathe K., Chapelle D., The finite element analysis of shells - fundamentals, Springer, 2003.

Berthelot J.-M., Materiaux composites - Comportement mecanique et analyse des structures, Masson, 1992.

Bihan D. L., Lepage A., Florens C., Roudolff F., Contrôle Actif de panneaux composites, Technical Report RT 1/08836 DDSS, Avril 2005.

Clouteau D., Arnst M., Al-Hussaini T., G. D., "Free field vibrations due to dynamic loading on a tunnel embedded in a stratified medium", Journal of Sound and Vibration, vol. 238, p. 173-199, 2005.

Craig R. J., "A Review of Time-Domain and Frequency Domain Component Mode Synthesis Methods", Int. J. Anal. and Exp. Modal Analysis, vol. 2, n 2, p. 59-72, 1987. 
Florens C., Balmes E., Clero F., Corus M., "Accounting for glue and temperature effects in Nomex based honeycomb models", ISMA, September, 2006.

Gibson L. J., Ashby M. F., Cellular Solids Structure and Properties, Second Edition, Cambridge University Press, 1997.

Grédiac M., "A finite element study of the transverse shear in honeycomb cores", International Journal of Solids and Structures, vol. 30(13), p. 1777-1788, 1993.

Hohe J., Becker W., "A refined analysis of the effective elasticity tensor for general cellular sandwich cores", International Journal of Solids and Structures, vol. 38, p. 3689-3717, 2001.

Jones R. M., Mechanics of Composite Materials, Hemisphere Publishing Corp., 1975.

Noor A., Burton W., "Assessment of continuum models for sandwich panel honeycomb cores", Computer methods in applied mechanics and engineering, vol. 145, p. 341-360, 1997.

Piefort V., Finite Element Modelling of Piezoelectric Active Structures, PhD thesis, Active Structures Laboratory Department of Mechanical Engineering and Robotics, Université Libre de Bruxelles, Belgium., 2001.

Sternchüss, A. and Balmes, E. and Jean, P. and Lombard, JP., "Reduction of Multistage disk models : application to an industrial rotor", 012502, 2008. Paper Number GT2008-012502. 


\section{ANNEXE POUR LE SERVICE FABRICATION \\ A FOURNIR PAR LES AUTEURS AVEC UN EXEMPLAIRE PAPIER \\ DE LEUR ARTICLE ET LE COPYRIGHT SIGNE PAR COURRIER LE FICHIER PDF CORRESPONDANT SERA ENVOYE PAR E-MAIL}

1. ARTiCle POUR LA REVUE:

RCMA - 19/2009. Composites multifonctionnels et réactifs

2. AUTEURS :

Etienne Balmes* - Corine Florens ${ }^{* * *}$

3. TITRE DE L'ARTICLE :

Validation of a vibration and electric model of honeycomb panels equiped with piezoelectric patch actuators

4. Titre abrégé Pour LE haUt de page MOINS De 40 Signes :

Honeycomb and piezo actuator model

5. DATE DE CETTE VERSiON :

December 7, 2009

6. CoOrdonnéES DES AUTEURS :

- adresse postale :

* Arts et Metiers ParisTech (LMSP)

151 Bld de l'Hopital, 75013 Paris

etienne.balmes@paris.ensam.fr

** ONERA DADS, 92322 Chatillon Cedex

Ecole Centrale Paris, MSSMat, 92295 Chatenay Malabry

- téléphone : 0144246371

- télécopie : 0000000000

- e-mail : etienne.balmes@paris.ensam.fr

7. LOGICIEL UTILISÉ POUR LA PRÉPARATION DE CET ARTICLE :

${ }_{\mathrm{AT}} \mathrm{X}$, avec le fichier de style article-hermes.cls, version $1.23 \mathrm{du} 17 / 11 / 2005$.

8. Formulaire DE COPYRIGHT :

Retourner le formulaire de copyright signé par les auteurs, téléchargé sur : http://www.revuesonline.com

SERVICE Éditorial - HERMES-LAVOISIER 14 rue de Provigny, F-94236 Cachan cedex Tél. : 01-47-40-67-67 E-mail : revues@lavoisier.fr 